\title{
Memories of the future
}

exposure to
cross-reactive
environmental
antigens may
drive the
development
of virus-
specific
memory T cells
in uninfected
individuals

0

It is generally believed that the development of pathogen-specific memory $\mathrm{T}$ cells requires previous exposure to the pathogen. However, a new study by Mark Davis and colleagues suggests that virus-specific memory-like $\mathrm{CD}^{+}{ }^{+} \mathrm{T}$ cells can be abundant in humans who have never previously been infected with, or vaccinated against, the virus in question.

The authors used tetramers specific for peptide epitopes presented on HLA-DR4 to identify rare, antigenspecific $\mathrm{CD} 4^{+} \mathrm{T}$ cells in the human $\mathrm{T}$ cell repertoire. They designed tetramers that could identify $\mathrm{T}$ cells specific for self-peptides (gp100, fibrinogen and preproinsulin) and also constructed tetramers that could identify $T$ cells specific for viral antigens (from HIV, herpes simplex virus (HSV), cytomegalovirus (CMV) and influenza virus). Using blood from 26 healthy human

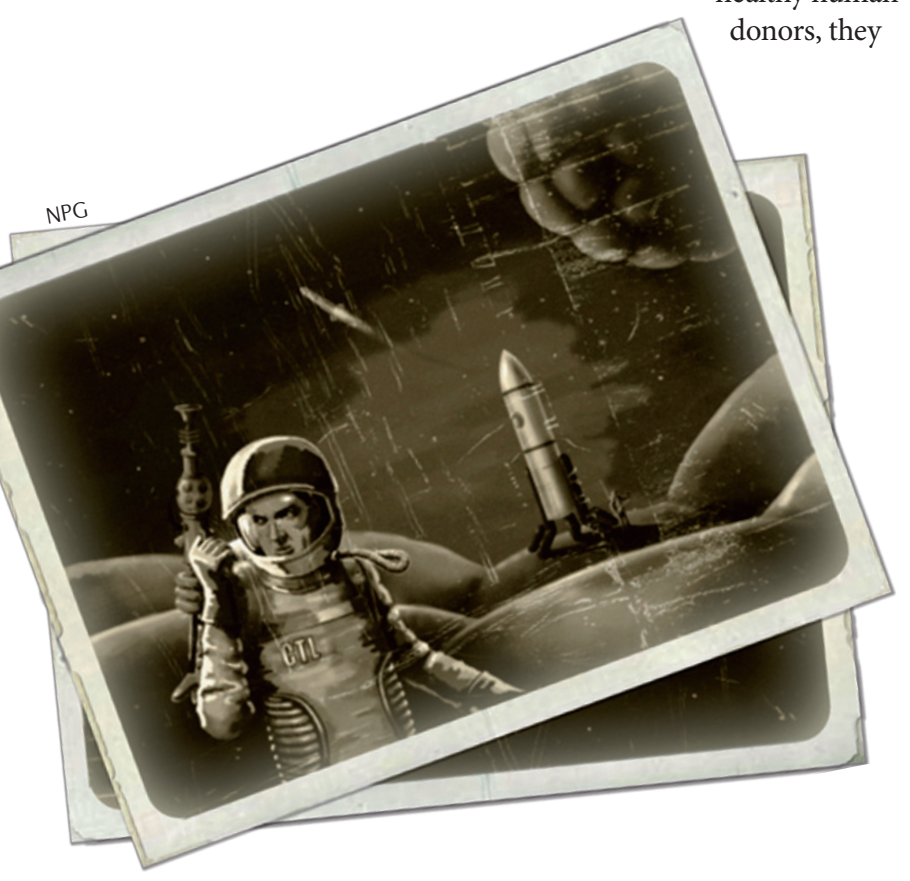

found that the frequency of $\mathrm{T}$ cells specific for a self antigen or for a viral epitope that had not previously been encountered ranged from one to ten cells per million $\mathrm{CD} 4^{+} \mathrm{T}$ cells. Notably, there was no obvious difference between the frequencies of $\mathrm{T}$ cells specific for self antigens and those specific for foreign peptides. In addition, similar frequencies of HSV-specific T cells were detected in individuals who were seropositive or seronegative for HSV, suggesting that pre-exposure to a particular infection does not necessarily lead to a detectable expansion in pathogen-specific T cells.

Next, the authors examined the memory phenotype of the tetramer-positive T cells. They were surprised to find many memorytype HIV-specific, CMV-specific and HSV-specific T cells in adults who were seronegative for these viruses - on average, more than $50 \%$ of the virus-specific T cells in these individuals showed a memory phenotype. When umbilical cord blood samples were assessed by tetramer analysis, the authors found similar frequencies of HIV-specific T cells to those seen in adults. However, unlike the adult HIV-specific T cells, all of the HIV-specific T cells in the cord blood samples showed a naive phenotype. So, newborns have a full repertoire of $\mathrm{T}$ cells that can recognize foreign antigens but do not acquire memory-like T cells until later in life.

The authors hypothesized that exposure to cross-reactive environmental antigens may drive the development of virus-specific memory $\mathrm{T}$ cells in uninfected individuals. To assess this, they generated distinct HIV-1-specific clones from human donors and stimulated them with peptides from environmental antigens that have a similar sequence to $\mathrm{T}$ cell epitopes present in the full-length HIV-1 peptide. They found that $21 \%$ of the HIV-1-specific clones responded to at least two of the putative cross-reactive peptides. Interestingly, the sources of the cross-reactive peptides were diverse and included bacteria from the intestine and soil, and also ocean algae and plants.

Finally, to study cross-reactive $\mathrm{T}$ cell responses to a known antigenic challenge, the authors vaccinated two individuals with an $\mathrm{H} 1 \mathrm{~N} 1$ influenza vaccine. They cloned $\mathrm{T}$ cells specific for haemagglutinin from this influenza virus strain and found that these $\mathrm{T}$ cells could also respond to peptides derived from the bacterium Finegoldia magna and the protozoan Trichomonas vaginalis. Furthermore, they showed that influenza vaccination not only expanded memory T cells specific for the seasonal haemagglutinin variant but also promoted the development of a population of memory-type $\mathrm{CD} 4^{+}$ $T$ cells that were also specific for the F. magna-derived peptide.

The authors suggest that a lack of these cross-reactive memory T cells in very young children could partly account for their vulnerability to infectious diseases. Furthermore, their findings provide a mechanism for studies showing that vaccination has survival benefits that are independent of protection from the specific pathogen targeted by the vaccine.

\section{Yvonne Bordon}

ORIGINAL RESEARCH PAPER Su, L. F. et al. Virus-specific $\mathrm{CD}^{+}$memory-phenotype T cells are abundant in unexposed adults. Immunity 7 Feb 2013 (doi:10.1016/j.immuni.2012.10.021) FURTHER READING Sewell, A. K. Why must

T cells be cross-reactive? Nature Rev. Immunol. 12, 669-677 (2012) 